\title{
Role of artificial intelligence in cloud computing, IoT and SDN: Reliability and scalability issues
}

\author{
Mohammad Riyaz Belgaum ${ }^{1}$ Zainab Alansari², Shahrulniza Musa ${ }^{3}$, Muhammad Mansoor Alam ${ }^{4}$, \\ M. S. Mazliham ${ }^{5}$ \\ ${ }^{1,3,4}$ Malaysian Institute of Information Technology, Universiti Kuala Lumpur, Malaysia \\ ${ }^{2}$ Faculty of Information Technology, Majan University College, Oman \\ ${ }^{2}$ Faculty of Computer Science \& Information Technology, University of Malaya, Malaysia \\ ${ }^{4}$ Institute of Business Management, Pakistan \\ ${ }^{5}$ Malaysia France Institute, Universiti Kuala Lumpur, Malaysia
}

\section{Article Info}

Article history:

Received Aug 9, 2020

Revised Dec 16, 2020

Accepted Jan 13, 2021

\section{Keywords:}

Artificial intelligence

Cloud computing

IoT

Reliability

Scalability

Software-defined networking

\begin{abstract}
Information technology fields are now more dominated by artificial intelligence, as it is playing a key role in terms of providing better services. The inherent strengths of artificial intelligence are driving the companies into a modern, decisive, secure, and insight-driven arena to address the current and future challenges. The key technologies like cloud, internet of things (IoT), and software-defined networking (SDN) are emerging as future applications and rendering benefits to the society. Integrating artificial intelligence with these innovations with scalability brings beneficiaries to the next level of efficiency. Data generated from the heterogeneous devices are received, exchanged, stored, managed, and analyzed to automate and improve the performance of the overall system and be more reliable. Although these new technologies are not free of their limitations, nevertheless, the synthesis of technologies has been challenged and has put forth many challenges in terms of scalability and reliability. Therefore, this paper discusses the role of artificial intelligence (AI) along with issues and opportunities confronting all communities for incorporating the integration of these technologies in terms of reliability and scalability. This paper puts forward the future directions related to scalability and reliability concerns during the integration of the above-mentioned technologies and enable the researchers to address the current research gaps.
\end{abstract}

This is an open access article under the CC BY-SA license.

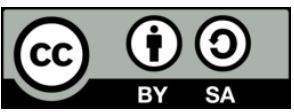

\section{Corresponding Author:}

Shahrulniza Musa

Malaysian Institute of Information Technology

Universiti Kuala Lumpur

1016 Jalan Sultan Ismail, 50250 Kuala Lumpur, Wilayah Persekutuan Kuala Lumpur, Malaysia

Email: shahrulniza@unikl.edu.my

\section{INTRODUCTION}

Artificial intelligence (AI) consciousness is playing a key role in changing the paradigm of every single noteworthy region of computing and innovation. AI works to create systems that can learn to mimic human behaviour from prior experience and without manual intervention. AI, which was once said to be rigid and a language of extreme computer intellectuals, has now become flexible and is being used in many areas [1]. AI has a lot of potentials, and what is being utilized at present is far less than what it has. AI reasoning enables various advancements by gathering the chronicled information, to play out a careful examination of them, distinguish the examples, and manual to make ongoing decisions. With the assistance 
of AI logic, it helps the consumer automate the method and increases knowledge by removing the plausibility of mistakes when performing the manual operation.

Industry 4.0 [2] supports the creation of a "smart factory" through process automation. The information technology field is commanded by a few different innovations, like cloud computing, the internet of things (IoT), and software-defined networking (SDN) with their enormous qualities in an interrelated space. However, there are various technologies supporting industry 4.0 as shown in Figure 1, contributing to the levels of productivity. AI with these technologies is at a revolutionary catalyst stage, where much attention is required. Artificial intelligence leads to cognitive intelligence through knowledge, understanding, and decision-making from past experiences. Artificial intelligence is speeding up with a high pace to achieve a technology that enables computers and machines to work smartly to allow the smart industry. The cloud services with IoT devices that can program following the wishes of each user are now on the brink of being satisfied with their performance. The heterogeneity [3] of the devices should allow them to communicate with each other with an interoperable data format. It leads to scalability and reliability with better user satisfaction. Reliability in this context is termed as the degree of trustworthiness by performing the joint services consistently and is an indicator stating what the systems percentage susceptibility to failure is. Scalability can be defined as the capacity of the system to match the increasing requirements comprehensively and allows the integration of growth. While here it is said to be the limit of the number of connected devices within a scenario in utilizing the resources efficiently. Scalability is the desired property of a service that enables the management of rising demand loads without substantial degradation of the related quality attributes [4].

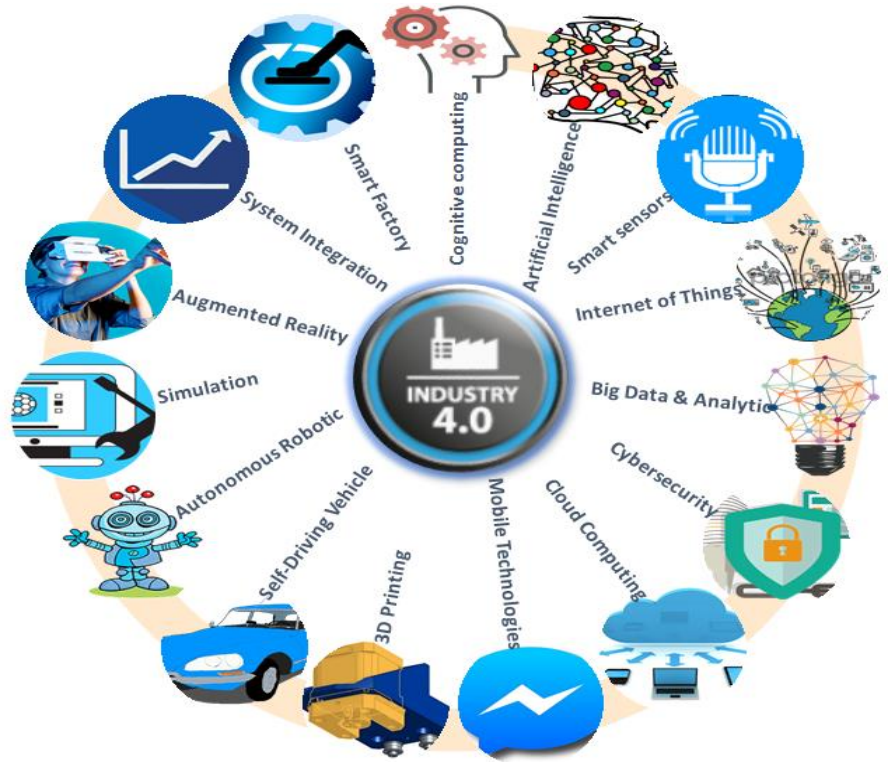

Figure 1. Technologies supporting Industry 4.0

The terms reliability and scalability are sometimes interdependent because the more the devices are connected and starve for the resources, the less is the reliability. On the other hand, if the number of connected devices is less, then most of the resources are left unused. So, to have proper utilization of the resources, AI should play a significant role by predicting the required usage of resources in advance. Various researchers are researching at integrating AI with cloud, AI with IoT and AI with SDN. But the integration of these emerging technologies with AI has still not been exposed, whose performance can be assessed using scalability and reliability attributes.

Across several diverse fields of technology [5], AI has powered recent developments and cloud computing, IoT and SDN are no exception. Though there are various other metrics used to assess the technologies reliability and scalability are more significant. Although various researchers have discussed the reliability and scalability issues in cloud computing, IoT and SDN separately but none have discussed the issues in the integration of these technologies. Moreover, this paper presents the reliability and scalability testing attributes which can be used in common to assess the performance of cloud computing, IoT and SDN. The role of AI in addressing some of the challenges of each of the above mentioned technologies are 
discussed. It also discusses the future directions which can be undertaken in the process of integrating the AI with mentioned three technologies.

The remaining part of the paper is discussed in the following sections after the introduction; Section 2 discusses the related literature. Section 3 focuses on the reliability and scalability testing attributes, section 4 discusses reliability and scalability in artificial intelligent cloud computing, artificial intelligent IoT, and artificial intelligent SDN. Then section 5 puts forward the research challenges and opportunities related to scalability and reliability during the fusion of the technologies mentioned above. Finally, section 6 discusses the conclusion.

\section{RELATED LITERATURE}

Different scholars have researched and analyzed the convergence of these technologies, and we present some relevant research in this segment. Reddy [6] the authors have discussed some of the challenges in the integration of IoT with wireless sensor networks. The challenges related to security, software and hardware have been discussed by the authors. However, the attributes used to access such integration have not been mentioned in it. The transformation from conventional to smart industry is done in many countries with the most significant attention. As in AlEnezi [7] survey, the authors demonstrate that mindscaping is Kuwait's primary challenge, investment is India's biggest challenge, and for the United States, security and privacy is the major problem to implement a smart-government. In a stable distributed infrastructure, the low power IoT devices should use the computing resources efficiently only when needed. So, Sharma [8] the authors' proposed controller fog nodes to respond to challenges such as usability, real-time data transmission, scalability, stability, durability and low latency at the edge of the scalable IoT networking using blockchainbased distributed cloud architecture with software-defined networking (SDN). In the dense wireless networks, an active device to device communication relays on cluster formation. The services provided by the cloud service providers can be assessed using quality of service (QoS) parameters. Gill and Buyya [9] discussed various open issues to be addressed like failure management, workload management, accountability, security, and physical infrastructure. Also, a conceptual model was proposed to match the dynamic requirements of the user with its reliable services. The communication among the IoT devices can be done using SDN and Hohlfeld [10] have put forth the challenges in data plane, control plane and application plane focusing on scalability. The challenges listed are traffic monitoring, plane interfaces and security including trust management and threat analysis.

Sharafeddine and Farhat [11] have proposed an approach to address reliable communication issues and considering the scalability of devices. A heuristic approach was used to select the cluster head and optimize the network reliability resulting in lower failure cost. In the field of electrical grids, the evolution of smart grid technology has enabled reliable power distribution. However, it is not free of attacks, and the anomaly detection technique proposed in [12] differentiates between a fault and disturbance and a cyberattack. symbolic dynamic filters (SDF) were used for the computer-efficient extraction of functions to identify causal correlations between the sub-systems of smart grids through dynamic bayesian networks (DBN). In high-speed networks where the attacks are increasing, the claim of authors stating the insufficiency of machine learning solutions for having a reliable intrusion detection is supported by the proposed approach big flow [13]. The proposed approach uses a verification model to prove its reliability by considering scalable network traffic with high accuracy over a period.

Rao et al. [14] have discussed a homomorphic encryption technique to provide enhanced security during the communication between cloud and consumer. In a smart weather system, the sensors are used to collect different types of values like humidity, temperature, raindrops, and $\mathrm{CO}_{2}$ emission. The values collected are stored in the cloud by avoiding storing in large databases. And to ensure the privacy of the data stored encryption is used. The stored data is accessed by various types of devices which increases the scalability. This data could be further analysed using any of the artificial intelligence technique for predicting the weather forecast. Plantevin et al. [15] presented an architecture for smart homes by adding intelligence in the transducer used for communication. The designed architecture collects raw data from the sensor and uses smart unit for communication with high reliability. The single point of failure was eliminated by using the proposed architecture and guaranteed scalability and reliability at a low cost. The attributes used to assess the reliability and scalability in each of the technology varies though some attributes are commonly used.

\section{RELIABILITY AND SCALABILITY ATTRIBUTES}

The attributes used to assess the reliability and scalability have a close relationship and dependability on each other. The following Figure 2 shows the list of attributes used to test reliability and scalability. The reliability of a system refers to the capacity of consistently fulfilling customer service 
requirements under the conditions of coexisting mitigating factors and increase in market volume [16]. The reliability of a system is a diverse device assessment issue in a multi-factor environment that has universality, heterogeneity, and uncertainty characteristics. The attributes used to test the reliability are explained in Table 1.

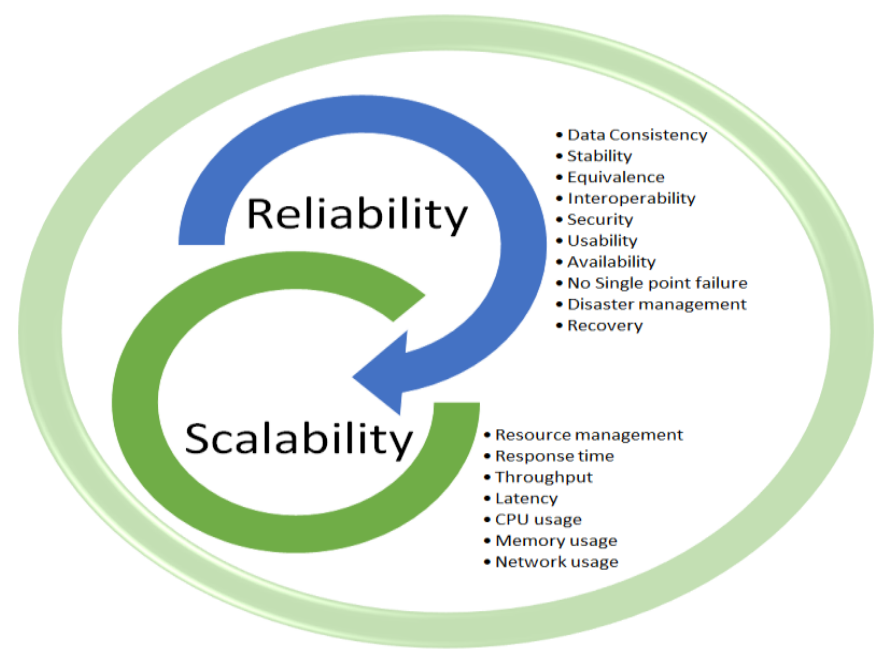

Figure 2. Reliability and scalability testing attributes

Table 1 . Reliability testing attributes

\begin{tabular}{ll}
\hline Attribute & Explanation \\
\hline Data & The extent to which all the data items on a scale measure one construct making the data consistent on all \\
Consistency & the devices. Consistency in data leads to better reliability. \\
Stability & The results obtained using an instrument should be consistent on repeated testing to ensure stability. \\
& And a highly stable system ensures reliability. \\
Equivalence & $\begin{array}{l}\text { Coherence between the responses of different device consumers or between the alternative type of } \\
\text { devices proves equivalence. Equivalence increases reliability. }\end{array}$ \\
Interoperability & $\begin{array}{l}\text { Through sharing data in a common standard format, the chosen device software will communicate with } \\
\text { on-site applications to allow the devices to interoperate. }\end{array}$ \\
Security & $\begin{array}{l}\text { The efficiency of data must be evaluated by the various organizations like NIST, COBIT and ISACA to } \\
\text { ensure security. The security is the most significant attribute as any security breach may result in }\end{array}$ \\
& $\begin{array}{l}\text { catastrophic incidences. } \\
\text { Thability }\end{array}$ \\
The usability term applies to the degree to which a particular form of the customer may utilize a device, \\
service or program to accomplish the final target with performance, efficiency and reliability while \\
interacting with various categories of computing devices. \\
Through sharing data in a common standard format, the chosen device software will communicate with \\
on-site applications to allow the devices to interoperate.
\end{tabular}

The concern to scalability is high in a centralized environment. Scalability is the ability to expand the computing service distribution capability by raising the amount of information delivered. Scalability is said to be provisioning and de-provisioning of devices [17]. It describes the load of the network as services are allocated. For scalable systems, the loading slope increases linearly with the increase of resources, while this line curve flattens out systems that do not size well. A segment between any two units that achieve maximum capacity with minimum deployment measures scalability. The attributes related to testing the scalability are discussed in Table 2.

\section{ROLE OF ARTIFICIAL INTELLIGENCE IN CLOUD COMPUTING, IOT AND SDN}

The emerging technologies discussed in this paper have various challenges to be addressed. AI plays an important role in addressing the challenges. The related literature studies are used to summarize some of the challenges considered in each of the technology as shown in Figure 3.

\subsection{Role of $\mathrm{AI}$ in cloud computing}

The incorporation of cloud computing into various online activities is already in progress [18]. The amalgamation of AI with cloud computing is significantly changing the way information technology (IT) is 
used across businesses and other industries with recent transformations. The various cloud computing services [19] play an essential role in the implementation of cloud-based solutions. The automated helpers such as Siri and Amazon Alexa from Apple are examples that changed our lives every day. The integration allows the cloud to be fitted as an artificial intelligence self-management. In IT systems, for example, AI after thorough analysis determines how to perform repetitive tasks using knowledge discovery. AI's ability to learn from past experiences allows the cloud to rebound from problems. The reliability of cloud services is increased as AI enhances data processing by efficiently storing and manipulating vast volumes of data. It enables data collection to be automated to provide customers with accurate information and triggering an alarm if the usual behaviour is aggressive. Intelligent analysis based on the modelling of deep neural networks can provide enterprises with much better control over their data and significant real-time impacts. AI gives consumers more interest in software-as-a-service (SaaS). Salesforce launched an AI tool called Einstein [20] which translates data into ideas. The tool uses customer data for its sales through advertisement and providing advice to customers by using various social networking means.

Table 2. Scalability testing attributes

\begin{tabular}{|c|c|}
\hline Attribute & Explanation \\
\hline $\begin{array}{l}\text { Resource } \\
\text { management }\end{array}$ & $\begin{array}{l}\text { Resources are very crucial and should be used efficiently and effectively. The increase in resources } \\
\text { increases the capacity of handling the load but the deallocation of resources should not affect the } \\
\text { performance of the system much. So, effective resource management supports the system in terms of } \\
\text { scalability. }\end{array}$ \\
\hline Response time & $\begin{array}{l}\text { The time from which the request has been made to the time the request started processing is said to be } \\
\text { response time. When a greater number of devices start using the same resource then the response time } \\
\text { decreases as the new requests will be waiting in the queue. }\end{array}$ \\
\hline Throughput & $\begin{array}{l}\text { The number of requests processed in a unit of time is called throughput. When multiple devices share a } \\
\text { common resource then the throughput decreases as the resource time has to be shared among all the devices. }\end{array}$ \\
\hline Latency & $\begin{array}{l}\text { Latency is the time delay taken to respond to the request. Latency can be due to setup time or } \\
\text { communication time too. }\end{array}$ \\
\hline CPU usage & $\begin{array}{l}\text { CPU is a very scarce resource and should be used very efficiently. When the scalability increases the CPU } \\
\text { usage also increases as a greater number of devices tend to use it. At the same time, the devices connected } \\
\text { should not fall below or exceed the threshold to have better CPU usage. }\end{array}$ \\
\hline Memory usage & $\begin{array}{l}\text { Increase in scalability occupies more memory. The devices information and the data from the devices are } \\
\text { stored in the memory. High scalability results in more memory usage. }\end{array}$ \\
\hline Network usage & $\begin{array}{l}\text { The devices are connected in a network and communicate with each other. The network usage is determined } \\
\text { by the link utilization between the devices. Increase in scalability increases network usage. }\end{array}$ \\
\hline
\end{tabular}

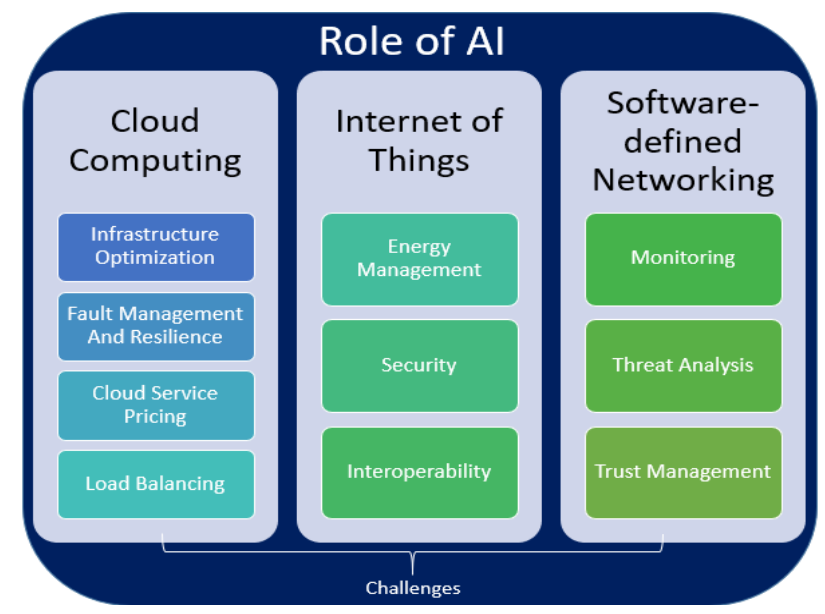

Figure 3. Role of AI in addressing challenges

Besides the services provided by the cloud to consumers, the use of artificial intelligence as an artificial intelligence-as-a-service (AIaaS) [21] is also available to the users. Various fields of AI like neural networks and machine learning, gather vast data sets to construct, train, process, and execute the models effectively. The analysis, calculation, and statistics of workload distributed over the cloud servers are, therefore, more readily accessible. Intelligently used are cloud resources, which can be dynamically extended up and down based on the tool. Manually processing huge volumes of data contributes to consumer loss over 
time. The artificial intelligence strongly supports fault tolerance [22] mechanism and tracks and handles application malfunction through the smooth relocation of servers. Supervised and unsupervised deep learning approaches are used to recognize and minimize loss trends in tandem with computational methods.

The integration of AI into cloud computing to make it accessible to everyone can be done by anchoring the cloud infrastructure with AI applications by continuous integration, continuous delivery, and phased deployments. A framework called model ops was proposed by the authors in [23] with reliable and trusted AI for cloud-based lifecycle management to reduce and manage the risks in deployment. The limitation of resource management under large scale use cases was left unaddressed. The software architects are thriving to develop a reliable and scalable cloud-assisted smart factory, which relates various AI technologies, and the logical relationship with different AI technologies. AI is powering the cloud to emerge as a self-managing cloud. Many researchers have speculated that when AI grows more advanced, private, and public clouds may rely on AI resources to track, control, and even self-heal in case of issue arises. Some of the major challenges in cloud computing to ensure reliability and support scalability along with the role of AI in providing solutions to them are listed in Table 3.

Table 3. Role of AI in addressing the challenges in cloud computing

\begin{tabular}{ll}
\hline $\begin{array}{l}\text { Major challenges in } \\
\text { cloud computing }\end{array}$ & Role of AI in providing a solution \\
\hline $\begin{array}{l}\text { Infrastructure } \\
\text { optimization }\end{array}$ & $\begin{array}{l}\text { Neural networks and fuzzy systems can be used for classification of operating status. } \\
\text { Machine language can be used for extracting the data from previous instances. } \\
\text { Evolutionary computing can be used for multi-objective optimization. }\end{array}$ \\
$\begin{array}{l}\text { Fault management } \\
\text { and Resilience }\end{array}$ & $\begin{array}{l}\text { Neural networks, fuzzy systems and evolutionary computing can be used for mapping the tasks } \\
\text { to the virtual machines. }\end{array}$ \\
Neural networks, fuzzy systems and machine learning can be used for prediction of the faults. \\
pricing & $\begin{array}{l}\text { Neural networks, fuzzy systems and machine learning can be used for predicting market price } \\
\text { trend and user demand. }\end{array}$ \\
& $\begin{array}{l}\text { Neural networks can be used for mapping cost and demand for a price. } \\
\text { Evolutionary computing can be used for multi-objective optimization. }\end{array}$ \\
Load balancing & $\begin{array}{l}\text { Neural networks can be used for detecting load imbalance and for mapping virtual machines } \\
\text { and dependability issues. }\end{array}$ \\
& Fuzzy systems can be used for specifying preferences, constraints, and management guidelines. \\
& Evolutionary computing can be used for multi-objective optimization.
\end{tabular}

\subsection{Role of $\mathrm{AI}$ in the internet of things}

The network of various objects linked through the internet captures and shares data without human intervention with one another is the internet of things [24]. The use of the internet to define, sense, link, and calculate personal, technical, and social artefacts has made life better and more difficult. All the IoT appliances produce a lot of data to obtain and use for operational performance. Artificial intelligence enters the picture here to capture and process the vast volume of data generated by the algorithms of artificial intelligence. Besides, these algorithms transform the data to achieve useful results that IoT devices can enforce. We have become almost knowledgeable by integrating artificial intelligence with those apps.

Figure 4 shows the process automation in areas such as the industrial internet of things (IIoT) and the consumer internet of things (CIoT), the introduction of artificial intelligence into IoT applications is relevant. For example, in [25], Z. Alansari the IoT plays a key role in the delivery of patient information to a doctor for sustainable development in the healthcare sector. The usage of IoT technologies has also extended into developing smart cities, smart homes, and to the broader context in smart buildings. The primary concern being automation of the operating of smart home devices using artificial intelligence and energy consumption optimization [26], many researchers have taken this as a challenge to gain user's satisfaction [27].

The increase of greed for comfort among the community is leading to access and share the information between the IoT, which in turn consumes more electricity and generate heat. Though there are many techniques to control energy consumption at households, they are still traditional. A framework has been proposed in [28] to predict the household energy consumption using feed-forward back propagation neural network to automate the process and ensure reliability. The goal of IoT is to provide the services under the limitation of low power long-range (LoRa) by ensuring reliability in communication. The authors in [29], B. Reynders focused LoRa wide-area networks (LoRaWAN) and proposed a two-step lightweight scheduling to improve the reliability and scalability. Each channel's transmission power and spreading factors are evaluated dynamically and the node decides onto which channel must be used for transmission. To ensure scalability the attribute used was throughput along with adding many nodes. And to ensure reliability the attributes used were packet error ratio and fairness. 
Under IoT, protection is a big issue, because most heterogeneous networks are related and interact with each other as they are scalable. There is still space for increasing bugs and attacks to infiltrate the network and carry out critical operations in this phase. Big data are complemented with the related network and social data, which are supplemented by different modes, which are focused upon physical, cyber social media (PCS). Artificial intelligence enables users to counter a range of attack styles, as defined in [30]. Some of the major challenges in IoT to ensure reliability and support scalability along with the role of AI in providing solutions to them are listed in Table 4.

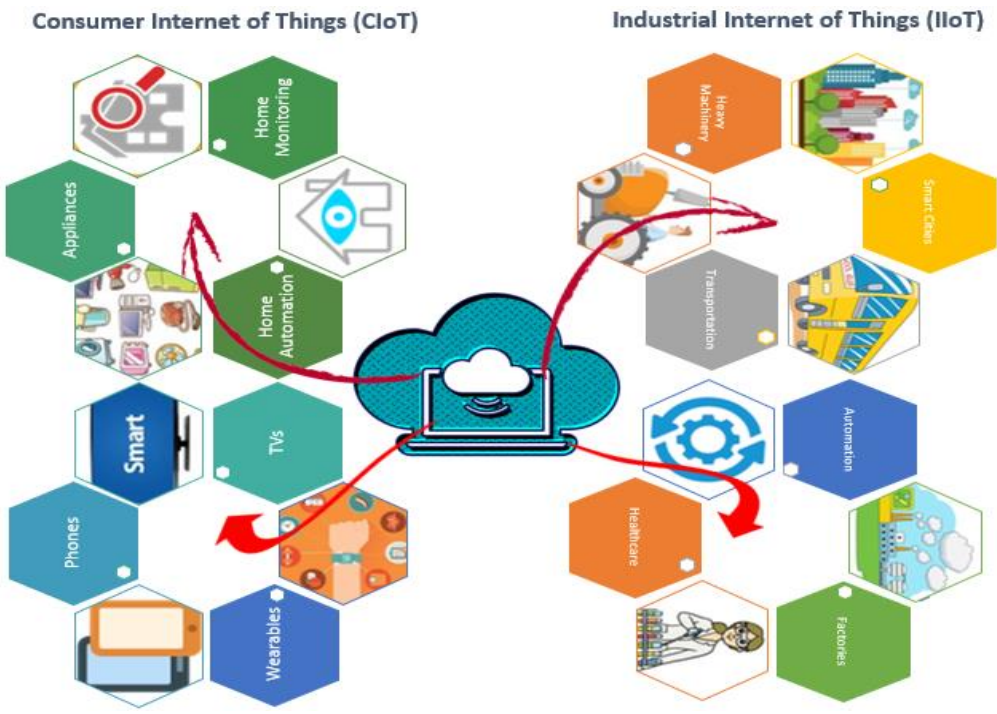

Figure 4. Consumer and Industrial IoT

Table 4. Role of AI in addressing the challenges in IoT

\begin{tabular}{|c|c|}
\hline Major challenges in IoT & Role of AI in providing a solution \\
\hline \multirow[t]{3}{*}{ Energy management } & Neural networks and fuzzy systems can be used for energy consumption prediction. \\
\hline & Neural networks can be used for classifying energy usage. \\
\hline & Machine language can be used for extracting the data from previous instances. \\
\hline Security & $\begin{array}{l}\text { Neural networks, fuzzy systems and machine learning can be used for classification and clustering } \\
\text { of security patterns in intrusion detection and malware detection. }\end{array}$ \\
\hline \multirow[t]{4}{*}{ Interoperability } & Fuzzy systems can be used for defining the matching preferences by specifying the constraints. \\
\hline & Machine learning can be used for extracting knowledge from previous instances. \\
\hline & Neural networks can be used for mapping the tasks. \\
\hline & Neural networks and fuzzy systems can be used for prediction of workload. \\
\hline
\end{tabular}

\subsection{Role of $\mathrm{AI}$ in Software-defined networking}

Software-defined networking has become a breakthrough in taking the network architectures to the next generation. This refines fine-grained flow management, which can configure and render networks to be more robust [31]. The decoupling of the control plane and data plane results in having a centralized view of the network with increased flexibility. The use of SDN in different areas like corporate and office networks, data centre networks, broad-based networks, and internet sharing centres made it possible to transit the conventional networking to SDN architecture. And this transition resulted in scalability issues because of the potential SDN switch flow data explosion. The minimal flow Table capacity of the SDN switch device significantly reduces the viability of large scale SDN deployment. The logical movement of the control planes from the switches to a centralized location has control over the entire network and has simplified the functionality but has also raised the issues on the scalability of the control plane as a result of virtualization.

The isolation of control plane and data plane from the switch and the concept of programming the network itself allows networking components produced by different vendors to be interoperable. The softwarization of networking in line with the three layers of SDN has become complicated due to the multilateral SDN domains in terms of reliability. In addition to artificial intelligence, SDN problems like scalability, efficiency, consistency, interoperability, and control, and protection overcome them and make the networking smart. There is little power on the ternary material addressable (TCAM) transition, and the 
controller changes the rules [32]. A rational device can adapt to potential inputs based upon past experiences. the gated recurrent recurrent neuro network (GRU-RNN) [33], is a supervised learning model used to solve security concerns by detecting intrusion, enabling the system to be more reliable. The error gradient is determined, and the activity of the components in the network is tracked to a minimum. The SDN reliability depends on the data plane reliability, control path reliability, and control plane reliability. Various authors have proposed solutions for issues on reliability. As part of them in [34], the authors have proposed a framework to address the issue of reliability and scalability with a case study. The framework included reduction of redundancy in the coupling of logical and control path along with enabling virtualization in the controller cluster, quick recovery of failures and orchestration of control messages to improve reliability and scalability. Some of the major challenges in cloud computing to ensure reliability and support scalability along with the role of $\mathrm{AI}$ in providing solutions to them are listed in Table 5.

Table 5. Role of AI in addressing the challenges in SDN

\begin{tabular}{ll}
\hline Major challenges in SDN & Role of AI in providing a solution \\
\hline Monitoring & Fuzzy systems can be used for specifying behaviour patterns and constraints. \\
& $\begin{array}{l}\text { Neural networks, Fuzzy systems and machine learning can be used for detecting behaviour } \\
\text { anomalies. }\end{array}$ \\
Threat analysis & $\begin{array}{l}\text { Neural networks, fuzzy systems and machine learning can be used for threat detection and } \\
\text { analysis. } \\
\text { Trust management }\end{array}$ \\
& $\begin{array}{l}\text { Neural networks, fuzzy systems and machine learning can be used for trust classification } \\
\text { and behaviour. }\end{array}$ \\
\hline
\end{tabular}

\subsection{Role of $\mathrm{AI}$ in the fusion}

The emerging technologies cloud, IoT and SDN have become game-changers in providing technical services to the end-users. And the fusion of AI and its synchronization with cloud computing, IoT and SDN is about to bring a significant change to the world of information technology and is going beyond the imagination by taking it to the next level. SDN appeared as a powerful platform to improve the scalability and versatility of IoT devices via the cloud. The progress in the field of communication, like the emergence of $5 \mathrm{G}$, has solved many issues of communications networking. Data collected from several IoT devices are gathered and accessible in a variety of formats and configurations to create massive data to be processed in the cloud and accessed via a vendor-free networking device. The IoT devices can exchange data with SDNs and cloud resources, even if the network is congested or network failure happens [35]. Artificial intelligence improves efficiency with its potential. The following Figure 5 indicates that artificial intelligence can be better integrated with various applications, even if its results are enhanced separately with cloud, IoT, SDN, and artificial intelligence convergence in a range of applications.

The above sections show that in tandem with each technology separately, artificial intelligence provides a better benefit. The ability to fuse artificial intelligence with all the discussed three technologies would enable societies to simplify operations in all aspects of digital technology and to meet the needs of industry 4.0. Artificial intelligence software-defined cloud of things (AISDCoT) allows the users to intelligently use the communication devices, and to securely transmit data, by implementing the SDN standards, through storing or accessing cloud-specified data.

The various streams of AI provide better solutions for various issues in each of the technology and have proved its effectiveness in many aspects by supporting in selection, allocation and mapping and optimization. The fuzzy systems can be used for defining the matching preferences, constraints in the cloud and specifying them to the connected devices via a network to attain a better solution. Fuzzy systems can also be used for classification and clustering of security patterns in the cloud, IoT devices as well as SDN to ensure reliability. After collecting the requirements from the users, the neural networks can be used to map the tasks to the virtual machines in the cloud and if load imbalance occurs, detection of load imbalance with consideration of dependability issues also can be done using neural networks. The classification of energy usage by the IoT devices connected can be done using fuzzy systems and neural networks to support scalability. The interconnection of devices and the connection with the cloud is done with networking capability. SDN enables to communicate with devices keeping them vendor-free and programming the network itself with multi-objective function. Evolutionary computing can be used to attain multi-objective optimization in the network considering the metrics like throughput, packet loss, delay, bandwidth utilization, and jitter contributing to improvising the quality of service. Machine learning is a subset of AI, which can be used for extracting knowledge from previous situations and predicting future occurrences. The following Figure 6 shows the interaction between each of the technology with the role of AI to provide reliable services to the end-user by using scalable devices. This Figure shows feasibility in the interaction of these technologies. 


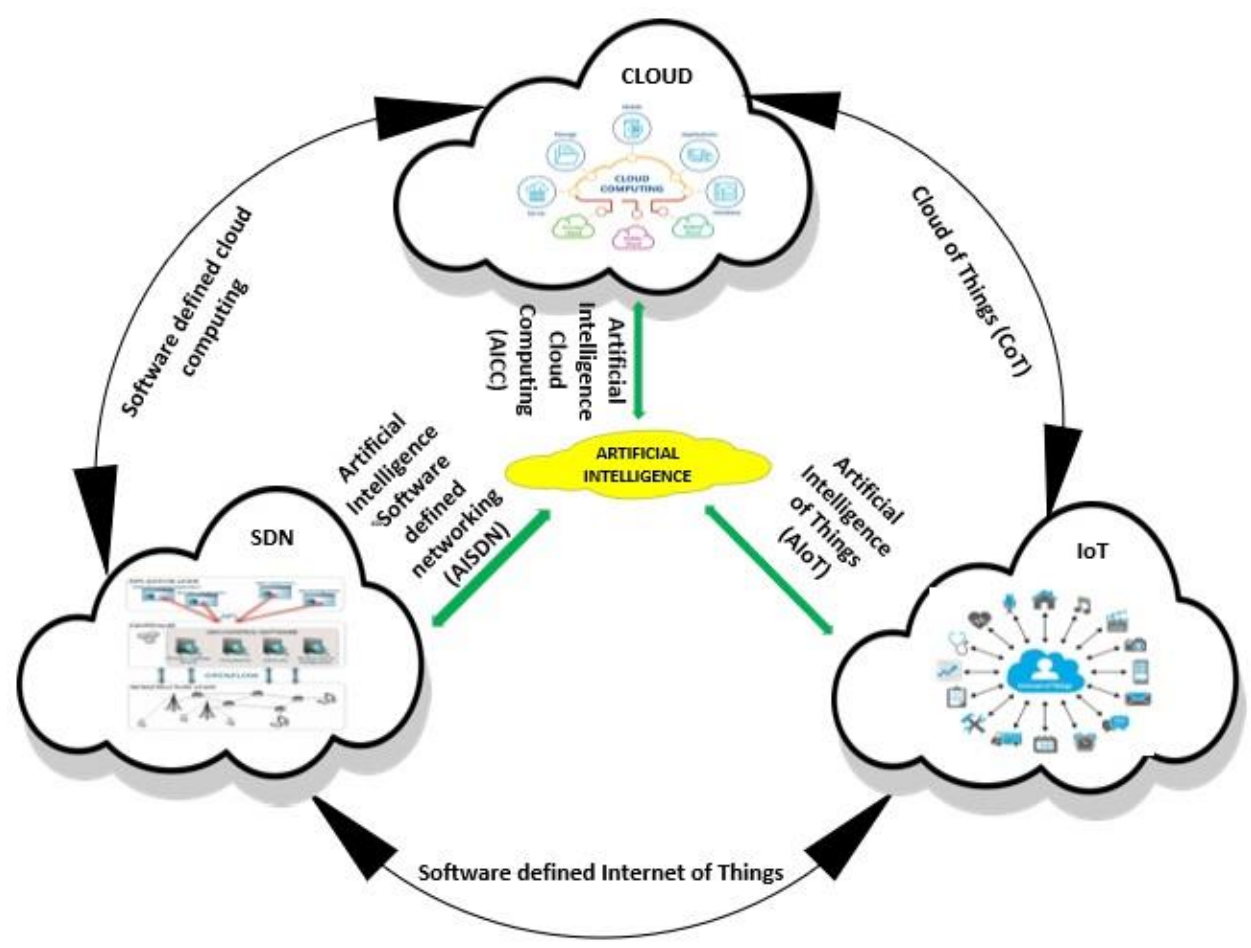

Figure 5. Artificial intelligence software-defined cloud of things (AISDCoT) [36]

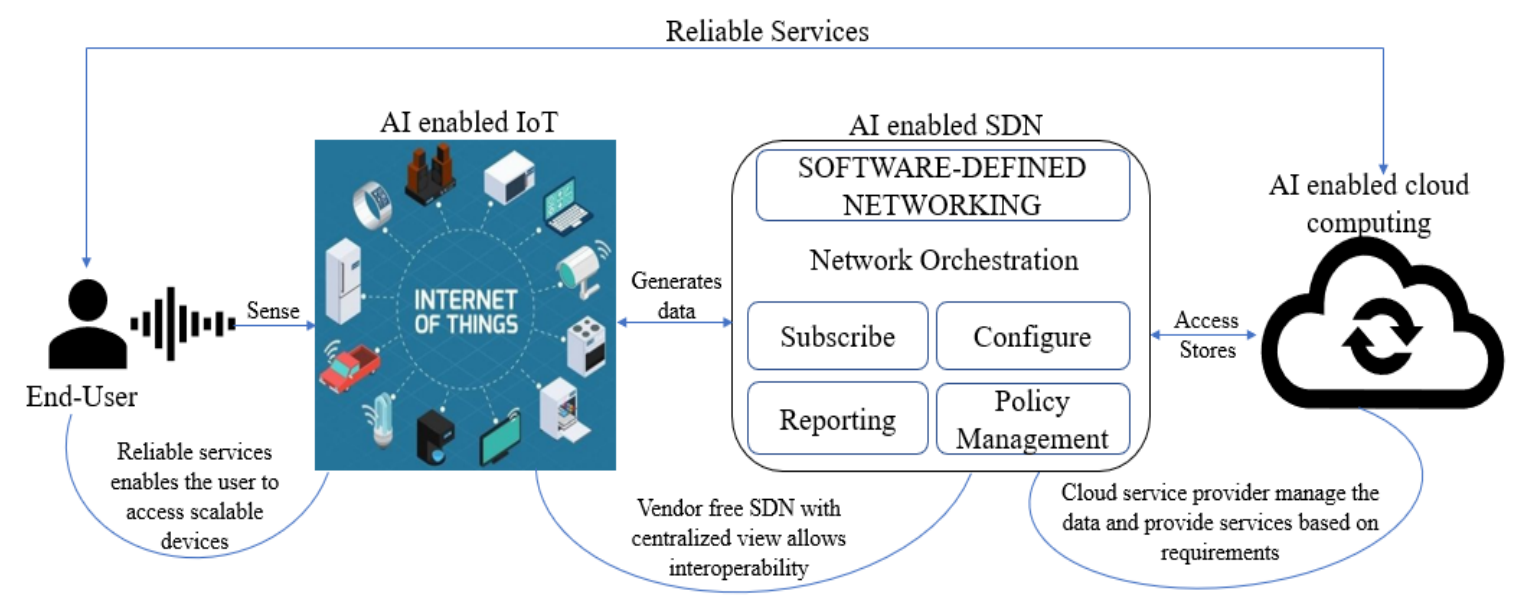

Figure 6. Reliability and scalability from the end-user's perspective

\section{FUTURE DIRECTIONS AND OPEN ISSUES}

The goal of every technology is to provide highly reliable and scalable services and products to the end-user, but occasional faults occur in any dynamic shared system. The following are some of the issues that can be considered as future directions of research while improving the reliability and scalability during the fusion of the above-mentioned technologies.

a. Security: The scalability of devices in the network may lead to an unreliable state if new devices are added and removed without authenticity. And if the smart devices are modular, they are very vulnerable to threats when these details are modified complex and often. So, as a future direction, an artificial intelligence technique can be developed to identify the authenticity along with preventing from different types of attacks to improve this incorporation.

b. Interaction: The IoT network interactions via SDN takes place among the devices, where the processing is done in the cloud. The end-user requirements can be met with the design of better architecture, protocols, 
and applications. Therefore, as a future direction, it is required to have efficient transmission by proposing using suitable artificial intelligence-capable techniques in designing protocols and applications for interaction.

c. Communication management: The devices communicate using various implementations but are restricted to a small network or specific functional jurisdiction. Large scale devices communication can cover large geographical areas, and such situations involve very flexible monitoring and management systems. Therefore, as a future direction, artificial intelligence traffic control and management are required to support reliable communication of a vast number of devices.

d. Flow Table update: SDN flow Tables have a minimum memory and can accommodate a couple of flow inputs. Frequent changing of flow Table entries results in extra overhead and becomes unreliable. So, as a future direction, there is a need for a mechanism, where artificial intelligence can be used to predict the flow Table entries and removal so that it makes the devices more scalable and reliable.

e. Interoperability: The quality of service depends on the interoperability of devices. And because various devices connect using different technologies, it lacks universal standards to lead. So, as a future direction, intelligent and comprehensive technology is therefore required to address the interoperability problem at all stages of communication.

f. Single point failure: Because of the modern and multi-lateral network environments, SDN becomes more complicated, to maintain the same continuity capabilities introduces several significant challenges to the existing network efficiency structures. While the controller allows operation and maintenance of a network incredibly easy for a network operator, the controller requires complex reasoning and becomes one point of failure within the network. Therefore, the artificial intelligence-enabled technique is needed to predict the failure, and measures are to be taken to avoid such failures, which is one of the future directions.

g. Programming: Consequently, the controller's design failures may cost the network provider incredibly much. Ultimately, one of the primary sources of this difficulty is a network failure, since they initiate the running of unexplored parts of code; such network errors are imminent. So, an efficient program should identify such failures and should predict before its occurrence from the past experiences, which is another future direction.

h. Load balancing: Wireless sensor devices communicate with each other and transmit the requests using the SDN interface. The protocol used for communication between the device and the control layer is the open flow protocol. The entries in the flow tables guide the order of execution. So, to dynamically allocate the load, an efficient artificial intelligence algorithm is needed. If a customized algorithm is built, the QoS can be improved.

i. Resource management: Efficient management of resources improves service efficiency parameters. The maintenance of resource usage statistics information is essential to keep track of and monitor the resources. As a future direction, the optimal resource management is to be done using an artificial intelligence technique to improve the service assessing metrics.

j. Energy efficiency: The major concern is to attain green computing and to enable so, heat pollution from several devices must be regulated. Artificial intelligence interference can help to conserve energy. This would mitigate and effectively reduce the energy consumption of these devices only when necessary. For many researchers, energy consumption reduction is an open issue and considered as a significant future direction to control the harmful heat generated by the machines.

\section{CONCLUSION}

This paper discusses the role of AI in cloud computing, IoT and SDN and raises a few issues regarding the integration of AI with cloud, IoT, and SDN, as these are today's current developments. The methods of artificial intelligence are used widely, which makes them work with little or no human intervention if these theories are paired with those patterns to function intelligently. Reliability and scalability testing attributes used to assess the performance have been discussed. The role of AI in addressing the challenges of cloud computing, IoT and SDN related to reliability and scalability have been discussed separately and in the integration of these technologies. Finally, the authors presented future directions and open issues to be addressed while merging the mentioned technologies. However, the training data issues, deployment issues, and computing performance have not been considered in the integration. In the process of solving the complexities of this fusion, the individual development problem will also be answered by resolving the common issues of all these phenomena. This study will support consumers and researchers who choose to perform more work on some of the above application combinations in the future. 


\section{REFERENCES}

[1] L. Tredinnick, "Artificial intelligence and professional roles," Business Information Review, vol. 34, no. 1, pp. 37-41, 2017, doi: 10.1177\%2F0266382117692621.

[2] S. Mittal, M. A. Khan, D. Romero, and T. Wuest, "A critical review of smart manufacturing and Industry 4.0 maturity models: Implications for small and medium-sized enterprises (SMEs)," Journal of manufacturing systems, vol. 49, pp. 194-214, 2018, doi: 10.1016/j.jmsy.2018.10.005.

[3] M. Noura, M. Atiquzzaman, and M. Gaedke, "Interoperability in internet of things: Taxonomies and open challenges," Mobile networks and applications, vol. 24, no. 3, pp. 796-809, 2019, doi: 10.1007/s11036-018-1089-9.

[4] S. Taherizadeh and V. Stankovski, "Dynamic multi-level auto-scaling rules for containerized applications," The Computer Journal, vol. 62, no. 2, pp. 174-197, 2019, doi: 10.1093/comjn1/bxy043.

[5] M. Saturno, V. M. Pertel, F. Deschamps, and E. de F. R. Loures, "Proposal of an automation solutions architecture for Industry 4.0," 24th International Conference on Production Research, Poznan, Poland, 2017.

[6] V. Reddy, "Integration of internet of things with wireless sensor network," International Journal of Electrical and Computer Engineering, vol. 9, no. 1, pp. 2088-8708, 2019, doi: 10.11591/ijece.v9i1.pp439-444.

[7] A. AlEnezi, Z. AlMeraj, and P. Manuel, "Challenges of IoT based Smart-government Development," $201821 s t$ Saudi Computer Society National Computer Conference (NCC), Riyadh, Saudi Arabia, 2018, pp. 1-6, doi: 10.1109/NCG.2018.8593168.

[8] P. K. Sharma, M. Chen, and J. H. Park, "A Software Defined Fog Node Based Distributed Blockchain Cloud Architecture for IoT," in IEEE Access, vol. 6, pp. 115-124, 2018, doi: 10.1109/ACCESS.2017.2757955.

[9] S. S. Gill and R. Buyya, "Failure Management for Reliable Cloud Computing: A Taxonomy, Model, and Future Directions," in Computing in Science and Engineering, vol. 22, no. 3, pp. 52-63, 1 May-June 2020, doi: 10.1109/MCSE.2018.2873866.

[10] O. Hohlfeld, J. Kempf, M. Reisslein, S. Schmid, and N. Shah, "Guest Editorial Scalability Issues and Solutions for Software Defined Networks," in IEEE Journal on Selected Areas in Communications, vol. 36, no. 12, pp. 2595-2602, Dec. 2018, doi: 10.1109/JSAC.2018.2872214.

[11] S. Sharafeddine and O. Farhat, "A proactive scalable approach for reliable cluster formation in wireless networks with D2D offloading," Ad Hoc Networks, vol. 77, pp. 42-53, 2018, doi: 10.1016/j.adhoc.2018.04.010.

[12] H. Karimipour, A. Dehghantanha, R. M. Parizi, K. R. Choo, and H. Leung, "A Deep and Scalable Unsupervised Machine Learning System for Cyber-Attack Detection in Large-Scale Smart Grids," in IEEE Access, vol. 7, pp. 80778-80788, 2019, doi: 10.1109/ACCESS.2019.2920326.

[13] E. Viegas, A. Santin, A. Bessani, and N. Neves, "BigFlow: Real-time and reliable anomaly-based intrusion detection for high-speed networks," Future Generation Computer Systems, vol. 93, pp. 473-485, 2019, doi: 10.1016/j.future.2018.09.051.

[14] Y. Narasimha Rao, P. S. Chandra, V. Revathi, and N. S. Kumar, "Providing enhanced security in IoT based smart weather system," Indonesian Journal of Electrical Engineering and Computer Science, vol. 18, no. 1, pp. 9-15, 2020, doi: 10.11591/ijeecs.v18.i1.pp9-15.

[15] V. Plantevin, A. Bouzouane, B. Bouchard, and S. Gaboury, "Towards a more reliable and scalable architecture for smart home environments," Journal of Ambient Intelligence and Humanized Computing, vol. 10, no. 7, pp. 2645-2656, 2019, doi: 10.1007/s12652-018-0954-5.

[16] N. Shaukat et al., "A survey on consumers empowerment, communication technologies, and renewable generation penetration within Smart Grid," Renewable and Sustainable Energy Reviews, vol. 81, pp. 1453-1475, 2018, doi: 10.1016/j.rser.2017.05.208.

[17] L. Bittencourt et al., "The internet of things, fog and cloud continuum: Integration and challenges," Internet of Things, vol. 3, pp. 134-155, 2018, doi: 10.1016/j.iot.2018.09.005.

[18] B. Varghese and R. Buyya, "Next-generation cloud computing: New trends and research directions," Future Generation Computer Systems, vol. 79, pp. 849-861, 2018, doi: 10.1016/j.future.2017.09.020.

[19] M. U. Bokhari, Q. Makki, and Y. K. Tamandani, "A survey on cloud computing," in Big Data Analytics, Springer, 2018, pp. 149-164, doi: 10.1007/978-981-10-6620-7_16.

[20] E. Turban, J. Outland, D. King, J. K. Lee, T.-P. Liang, and D. C. Turban, "Intelligent (smart) E-commerce," in Electronic Commerce 2018, Springer, 2018, pp. 249-283, doi: 10.1007/978-3-319- 58715-8_7.

[21] S. A. Javadi, R. Cloete, J. Cobbe, M. S. A. Lee, and J. Singh, "Monitoring Misuse for Accountable'Artificial Intelligence as a Service," in Proceedings of the AAAI/ACM Conference on AI, Ethics, and Society, 2020, pp. 300-306, doi: 10.1145/3375627.3375873.

[22] J. Lee and J. Gil, "Adaptive fault-tolerant scheduling strategies for mobile cloud computing," The Journal of Supercomputing, vol. 75 , no. 8, pp. 4472-4488, 2019, doi: 10.1007/s11227-019-02745-5.

[23] W. Hummer, et al., "ModelOps: Cloud-Based Lifecycle Management for Reliable and Trusted AI," 2019 IEEE International Conference on Cloud Engineering (IC2E), Prague, Czech Republic, 2019, pp. 113-120, doi: 10.1109/IC2E.2019.00025.

[24] J. I. R. Molano, J. M. C. Lovelle, C. E. Montenegro, J. J. R. Granados, and R. G. Crespo, "Metamodel for integration of internet of things, social networks, the cloud and industry 4.0," Journal of ambient intelligence and humanized computing, vol. 9, no. 3, pp. 709-723, 2018, doi: 10.1007/s12652-017-0469-5.

[25] Z. Alansari, S. Soomro, M. R. Belgaum, and S. Shamshirband, "The rise of Internet of Things (IoT) in big healthcare data: review and open research issues," in Progress in Advanced Computing and Intelligent Engineering, Springer, 2018, pp. 675-685, doi: 10.1007/978-981-10-6875-1_66.

[26] M. M. Tumari, M. H. Suid, and M. A. Ahmad, "A modified Grey Wolf Optimizer for improving wind plant energy 
production," Indonesian Journal of Electrical Engineering and Computer Science, vol. 18 no. 3, 1123-1129, 2020, doi: 10.11591/ijeecs.v18.i3.pp1123-1129.

[27] I. O. Essiet, Y. Sun, and Z. Wang, "Optimized energy consumption model for smart home using improved differential evolution algorithm,” Energy, vol. 172, pp. 354-365, 2019, doi: 10.1016/j.energy.2019.01.137.

[28] M. Fayaz, H. Shah, A. M. Aseere, W. K. Mashwani, and A. S. Shah, "A framework for prediction of household energy consumption using feed-forward back propagation neural network,” Technologies, vol. 7, no. 2, p. 30, 2019, doi: 10.1155/2019/8640218.

[29] B. Reynders, Q. Wang, and P. Tuset-Peiro, X. Vilajosana and S. Pollin, "Improving Reliability and Scalability of LoRaWANs Through Lightweight Scheduling," in IEEE Internet of Things Journal, vol. 5, no. 3, pp. 1830-1842, June 2018, doi: 10.1109/JIOT.2018.2815150.

[30] L. Xiao, X. Wan, X. Lu, Y. Zhang, and D. Wu, "IoT Security Techniques Based on Machine Learning: How Do IoT Devices Use AI to Enhance Security?," in IEEE Signal Processing Magazine, vol. 35, no. 5, pp. 41-49, Sept. 2018, doi: 10.1109/MSP.2018.2825478.

[31] M. R. Belgaum, S. Musa, M. M. Alam, and M. M. Su'ud, "A systematic review of load balancing techniques in softwaredefined networking," in IEEE Access, vol. 8, pp. 98612-98636, 2020, doi: 10.1109/ACCESS.2020.2995849.

[32] C. Chuang, Y. Yu, and A. Pang, "Flow-Aware Routing and Forwarding for SDN Scalability in Wireless Data Centers," in IEEE Transactions on Network and Service Management, vol. 15, no. 4, pp. 1676-1691, Dec. 2018, doi: 10.1109/TNSM.2018.2865166.

[33] T. A. Tang, L. Mhamdi, D. McLernon, S. A. R. Zaidi, and M. Ghogho, "Deep Recurrent Neural Network for Intrusion Detection in SDN-based Networks," 2018 4th IEEE Conference on Network Softwarization and Workshops (NetSoft), Montreal, QC, Canada, 2018, pp. 202-206, doi: 10.1109/NETSOFT.2018.8460090.

[34] S. Song, H. Park, B. Choi, T. Choi, and H. Zhu, "Control Path Management Framework for Enhancing SoftwareDefined Network (SDN) Reliability," in IEEE Transactions on Network and Service Management, vol. 14, no. 2, pp. 302-316, June 2017, doi: 10.1109/TNSM.2017.2669082.

[35] K. E. Benson, G. Wang, N. Venkatasubramanian and Y. Kim, "Ride: A Resilient IoT Data Exchange Middleware Leveraging SDN and Edge Cloud Resources," 2018 IEEE/ACM Third International Conference on Internet-ofThings Design and Implementation (IoTDI), Orlando, FL, USA, 2018, pp. 72-83, doi: 10.1109/IoTDI.2018.00017.

[36] M. R. Belgaum, S. Musa, M. Alam and M. S. Mazliham, "Integration challenges of Artificial Intelligence in Cloud Computing, Internet of Things and Software-defined networking," 2019 13th International Conference on Mathematics, Actuarial Science, Computer Science and Statistics (MACS), Karachi, Pakistan, 2019, pp. 1-5, doi: 10.1109/MACS48846.2019.9024828.

\section{BIOGRAPHIES OF AUTHORS}

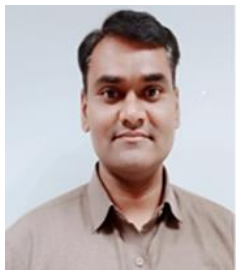

Mohammad Riyaz Belgaum received his master's degree with a specialization in Computer applications in 2001 and a Master of Engineering with a specialization in Computer Science and Engineering in 2006. He is an Oracle Database 11G Administrator Certified Professional and an Oracle Database 11G Program with PL/SQL Certified Associate. He is currently pursuing his $\mathrm{PhD}$ in Information Technology at Universiti Kuala Lumpur with a focus on Software Defined Networks. His areas of interest include cloud computing, mobile ad hoc networking, wireless sensor networks, Internet of Things, and bytecode analysis. He has authored more than 30 research articles published in journals and peer-reviewed conferences. He has vast teaching experience across various countries with much focus on research.

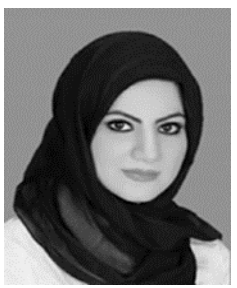

Zainab Alansari received her B.S. degree in Computer Science with summa cum laude award in 2007 and M.S. degree in Computer System and Networking with the highest honour degree award and the best thesis award in 2011, both from the AMA International University, Bahrain. Since 2007, she has been a Lecturer and Instructor of Computer Science in several institutions internationally. Zainab Alansari published more than 35 ISI and Scopus-indexed articles and contributed to numerous conferences and seminars internationally as a presenter, keynote speaker and session chair. Her research interests include the Internet of Things, Wireless Sensor Networks, Big Data and Cloud Computing. She is currently a senior lecturer in Majan University College, Muscat, Oman and completing her PhD in Computer Science from the University of Malaya (UM) in Malaysia.

Shahrulniza Musa is the Deputy President in charge of the Academic and Technology of Universiti Kuala Lumpur (UniKL). He started his career at UniKL since its establishment in 2002. He received his Post-Graduate Diploma in Integrated Research Study in 2005 and Doctor of Philosophy $(\mathrm{PhD})$ in Communication Network Security in 2008, both from the Faculty of Electrical and Electronic Engineering, Loughborough University, UK. His research interest is in Cybersecurity, IoT application, IoT security, BigData Analytics and SDN. Apart from teaching and post-graduate supervision, he is also active in Software project consultation and development in Business Application, Enterprise Resource Planning (ERP) and customer relation management (CRM). His ORCID ID and SCOPUS ID are 0000-0003-4867-5085 and 52963995100, respectively. 

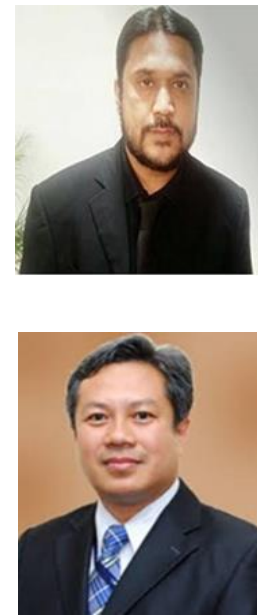

Muhammad Mansoor Alam received an M.E. degree in systems engineering, an M.Sc. degree in computer science, a $\mathrm{PhD}$ degree in computer engineering, and a $\mathrm{PhD}$ degree in electrical and electronic engineering. He is an active researcher in the field of telecommunication and network. He has authored more than 60 research articles published in ISI indexed journals, as book chapters, and in peer-reviewed conferences. He is also an author of the book "Study Guide of Network Security" copyrighted by Open University Malaysia and The Open University of Hong Kong. He is also an active reviewer for the ISI indexed journal Pertanika Journal of Science and Technology (JST).

Mazliham Mohd Su'ud received a PhD degree in computer engineering from the Université de La Rochelle, in 2007 and a master's degree in electrical and electronics engineering from the University of Montpellier, in 1993. He has been the President/CEO of Universiti Kuala Lumpur, Malaysia for seven years from 2013. Currently, he is the President/CEO of Multimedia University, Malaysia. 